\title{
EFECTO DE LA APLICACIÓN DE UNA MULTIMEDIA EN LA ADQUISICIÓN DE DESTREZAS PARA LA EVALUACIÓN DE COMPONENTES DE LA APTITUD FÍSICA RELACIONADOS A LA SALUD, EN ESTUDIANTES DE EDUCACIÓN FÍSICA DE LA UNIVERSIDAD NACIONAL
}

\author{
Irina Anchía Umaña ${ }^{1}$, Gerardo A. Araya Vargas ${ }^{1 y 2}$ y Juan Carlos \\ Gutiérrez Vargas ${ }^{1}$. \\ ${ }^{1}$ Escuela de Ciencias del Movimiento Humano y Calidad de Vida, \\ Facultad de Ciencias de la Salud, Universidad Nacional de Costa Rica \\ ${ }^{2}$ Escuela de Educación Física y Deportes, Facultad de Educación, \\ Universidad de Costa Rica \\ ianchia@una.cr
}

RESUMEN

\begin{abstract}
El objetivo de este estudio fue determinar el efecto de la aplicación de una multimedia en la adquisición de destrezas necesarias en la evaluación de componentes de la aptitud física relacionados a la salud. Metodología: sujetos: estudiantes universitarios de educación física ( $\mathrm{n}=29$ ), matriculados en el curso de Evaluación de Cualidades Físicas. Instrumentos: multimedia de batería de pruebas de evaluación física relacionadas a la salud (pliegues subcutáneos, capacidad cardiorrespiratoria, flexibilidad, fuerza muscular y resistencia muscular abdominal modificada), software de autoría Neobook para elaboración de publicaciones electrónicas, versión 5.0; software para el desarrollo de evaluaciones académicas Hot Potatoes de Half - Baked Software Inc, versión 6; Adobe Premiere para capturar y editar video, versión Pro 1.5; Audicity para grabación de audio; programa básico para la edición de texto Word, cámara de video DCR-VX2000 SONY en formato MiniDV NTSC, para la grabación de video. Procedimiento: los sujetos realizaron el primer test (aplicación de pruebas para evaluar componentes de aptitud física relacionados con la salud) y tuvieron un evaluador ciego y con base en los resultados de este pretest, se distribuyeron en tres grupos de tratamiento, por la modalidad de puntajes aparejados y a cada grupo, se le aplicó un tratamiento diferente: solo teoría, solo multimedia y teoría - multimedia. Cada grupo recibió 1 hora de tratamiento por 10 semanas y finalizado este proceso, el evaluador ciego procedió a aplicar el post test para evaluar componentes de aptitud física relacionados con la salud a los 3 grupos. Asimismo se realizó una entrevista de grupo focal a cada grupo por separado, donde se identificaron factores cualitativos. Análisis Estadístico: Se utilizó un ANOVA de dos vías mixta (3 tratamientos x 2 mediciones) para el análisis cuantitativo y el análisis de contenido y categorización para los datos cualitativos. Resultados: Se encontró que no hubo interacción significativa entre mediciones y grupos, ni diferencias significativas entre grupos. Aunque se pudo observar una tendencia positiva pero no significativa en el grupo mixto (teoría-multimedia), el cual obtuvo puntajes mayores que los otros grupos. Por otro lado, sí se encontraron diferencias estadísticamente significativas entre las mediciones, es decir todos los grupos mejoraron los puntajes en la segunda medición. En la entrevista se puede observar una tendencia de criterio positiva hacia el uso de la multimedia como herramienta de refuerzo para mejorar los procesos de enseñanza a nivel de educación superior y específicamente en el área de las ciencias del movimiento humano y la salud. Discusión: En términos generales los resultados estadísticos indican que usar un multimedia, un multimedia como ayuda a un profesor y un profesor sin ayuda multimedial, para generar conocimiento en el área de la evaluación física no presenta diferencias, y que los tres tipos de procesos pueden ser igualmente exitosos mientras se mantengan condiciones como las presentadas en esta investigación. Por otro lado, los resultados de la entrevista permiten observar que en general se acepta la multimedia como un elemento que ayuda a mejorar el proceso de aprendizaje en el aula. Conclusiones: la multimedia funciona como un elemento que sirve para ayudar en los procesos de enseñanza en la transmisión de información técnica, permitiéndole al profesor dedicar más tiempo a otras actividades.
\end{abstract}

PALABRAS ClAVES: Tecnologías Educativas de la Información y Comunicación, Multimedia, Procesos Académicos, Docente, Componentes de aptitud física, Protocolo de evaluación física, Procesos de aprendizaje, Tecnologías, Modelos de aprendizaje, Procesos académicos, Evaluación de la aptitud física, Herramientas para el aprendizaje. 


\begin{abstract}
The objective of this research was to determine the effect of the application of a multimedia in the acquisition of necessary skills in the evaluation of the physical fitness components related to health. METHODOLOGY: subjects: Physical college students $(\mathrm{n}=29)$, enrolled in Physical Qualities Evaluation course. Instruments: multimedia of battery of physical evaluation proof related to health ( subcutaneous wrinkles, cardio respiratory capacity, flexibility, muscle strength, abdominal muscular resistence modified), software of Neobook Authorship for the elaboration of electronic publications, version 5.0: software for the academic evaluations development Hot Potatoes of Half- Baked Software Inc., version 6, Adobe Premiere to capture and edit video version Pro 1.5, audacity of audio recording, basic program for the word text edition, video camera DCR- VX 2000 Sony in MiniDVNTSC format, for the video recording. Procedure: the subjects accomplished the first test ( test application to evaluate the physical ability components related to health) and they had a blind assessor and with the basis of the results of this pretest, it was arranged into three treatment groups, about the linked score modality, and each group got a different treatment: just theory, just multimedia and theory-multimedia. Each group received 1 hour of treatment for 10 weeks, when this process was finished, the blind assessor proceeded to apply the post test to evaluate the physical ability components related to health to the three groups. Additionally a focal group interview was carried out to each group separately, where qualitative factors were identified. Statistical Analysis: ANOVA of two mixed vias was used ( 3 treatments x 2 measuring) for the quantitative analysis and the content analysis and for the qualitative datum categorization. Results: It was found that there was not any meaningful interaction between measuring and groups, neither meaningful differences between groups. Although it could be observed a positive tendency but not meaningful in the mixed group (theory-multimedia) which had larger score than the other groups. In the other hand, it was found differences statistically meaningful between the measuring in other words, every group improved the score on the second measurement. In the interview it could be seen a tendency of positive criteria toward the use of multimedia as a reinforcement tool to improve the teaching process at the level of college education specifically in the science of human movement and health area. Discussion: By and large the statistical results show that the use of multimedia, multimedia as a professor help and a professor without multimedia help to create knowledge in the physical evaluation area; they present no differences, and the three types of processes can be equally successful meanwhile there are kept the conditions as the ones proposed in this research. On the other hand, the results of the interview let it note that multimedia in general is accepted as an element that help to improve the learning process in the classroom. Conclusions: Multimedia works as an element that helps in the learning process in the transition of technical information, allowing the professor to spend time in other activities.
\end{abstract}

KEY WORDS: Information and Communication Educational Technologies, Multimedia, Academic Process, Professor, Physical Fitness, Physical Evaluation Protocol, Learning process, Technologies, Learning Models, Academic Process, Physical Fitness Evaluation, Learning Tools.

\title{
INTRODUCCIÓN:
}

El desarrollo de los microordenadores y la tecnología multimedia ha llamado de inmediato la atención de la comunidad universitaria y educativa en general, la cual necesita de estos sistemas de procesamiento de información adecuados para un aumento exponencial del conocimiento. Por este motivo desde la década de los años ochenta, el hipertexto y las multimedia son parte integrante, aunque marginal, de los proyectos de modernización y actualización experimental en el diseño de las políticas de planeación educativa en numerosas universidades, abriendo así la puerta a una infinidad de problemas aún no suficientemente investigados (Sierra, 2002).

Hasta el momento existe mucha tecnología aplicada a los procesos de enseñanzaaprendizaje, pero en su mayoría, estos procesos se quedan en un plano teórico y difícilmente van a la aplicación práctica, otros van a la práctica sin la necesaria evaluación teórica y lo que es peor, sin el acompañamiento de la investigación que permita orientar los procesos. Costa Rica no es la excepción a esta situación, y es hasta hace pocos años que se comienzan a hacer esfuerzos para el estudio práctico de la aplicación de las tecnologías (Álvarez y Hernández, 2005). 
Es importante entender cuáles son los procesos necesarios para que la tecnología pueda ser aprovechada al máximo y en este sentido existe gran desconocimiento, más si se toma en cuenta que cada día los cambios tecnológicos se aceleran más y más, lo que implica una aparición constante de nuevas tecnologías que pueden hacer obsoletos los conocimientos que se tenían ayer. Nace de esto la necesidad de una constante investigación que indique el camino a seguir con respecto a la aplicación educativa (Álvarez y Hernández, 2005).

\section{METODOLOGÍA}

Sujetos: participaron como sujetos de investigación 29 estudiantes de la carrera de Bachillerato en la Enseñanza de la Educación Física, Deporte y Recreación de la Escuela de Ciencias del Movimiento Humano y Calidad de Vida (CIEMHCAVI) de la Universidad Nacional (UNA); 14 hombres y 15 mujeres, con una edad promedio de 20.3 años \pm 5.4 , distribuidos de la siguiente manera: grupo teoría $=3$ hombres y 6 mujeres; grupo multimedia $=6$ hombres y 8 mujeres; grupo multimedia-teoría $=5$ hombres y 1 mujer.

Instrumentos: se aplicó un multimedia (su proceso de elaboración se detalla más adelante) de una batería de pruebas de evaluación física relacionadas a la salud, cuyas pruebas físicas incluidas eran: pliegues subcutáneos, capacidad cardiorrespiratoria, flexibilidad, fuerza muscular y resistencia muscular abdominal modificada. También se usó varios software: uno de autoría Neobook para elaboración de publicaciones electrónicas, versión 5.0; software para el desarrollo de evaluaciones académicas Hot Potatoes de Half - Baked Software Inc, versión 6; Adobe Premiere para capturar y editar video, versión Pro 1.5; Audicity para grabación de audio; programa básico para la edición de texto Word. También se usó una cámara de video DCR-VX2000 SONY en formato MiniDV NTSC, para la grabación de video.

Procedimiento: para la elaboración de la multimedia sobre pruebas de evaluación física, se procedió a la búsqueda de información de esta materia y de ahí específicamente sobre componentes de aptitud física relacionados a la salud. Una vez concluida la búsqueda, se siguió con la selección de los protocolos que se incluyeron dentro de la multimedia y cada uno de ellos con su respectivo guión técnico y guión literario; se prepararon los materiales y el ambiente en el cual serían grabados los videos, luego de haberse concluido con la grabación de estos, se procedió a elaborar la multimedia utilizando los materiales descritos en el apartado de instrumentos.

Posterior a esto se procedió a hacer solicitud de los debidos permisos a la Escuela, para el uso del laboratorio de cómputo, del aula número 1 y del aula número 2 , para aplicar cada uno de los tratamientos y para colocar los afiches del curso en lugares visibles de la Escuela. Se publicó el anuncio sobre el curso de Evaluación de Componentes de Aptitud Física relacionados a la salud y luego se realizó la inscripción de los estudiantes al curso. La forma en la que se atrajo a los estudiantes a parte del anuncio que se publicó, fue que se 
les ofreció un certificado de participación de 20 horas, se les obsequió una multimedia de masaje, una de ejercicios de piso y la de evaluación de componentes de aptitud física (la que se elaboró para el curso propiamente).

Antes de dar inicio al curso, se realizó un pre test aplicado por dos evaluadores expertos en el área de evaluación física, mediante el método de evaluador ciego, esto quiere decir que los evaluadores no tenían conocimiento durante la investigación, de cuáles sujetos estaban en cuál de los grupos de tratamiento, con el fin de determinar cuánto conocimiento poseían los y las estudiantes acerca de la evaluación de componentes de aptitud física relacionados con la salud y su adecuada ejecución de la técnica con la mayor imparcialidad.

Dicho pre test fue aplicado en el laboratorio de evaluación fisiológica del Programa de Ciencias del Ejercicio y la Salud (PROCESA) de CIEMHCAVI dado que ahí se contada con la facilidad de tener a mano los materiales e instrumentos necesarios para efectuar las evaluaciones. Se dividió el grupo en dos para que ambas partes fueran evaluadas por un evaluador ciego cada una. Después de realizar esta división se convocó a los estudiantes a una hora determinada y se les solicitó que llegaran en parejas para que se evaluaran entre ellos para mayor facilidad.

Con base en los resultados obtenidos en el pre test, se elaboró una lista por orden de nota de mayor a menor y tomándola como referencia, se distribuyó a los y las estudiantes en tres grupos de tratamiento por la modalidad de puntajes aparejados.

Finalizado lo anterior, se procedió a iniciar con cada uno de los tratamientos que consistió en lo siguiente:

El primer grupo se denominó Grupo Teoría (T) y trabajó asistido por la guía de la investigadora utilizando el método de clase tradicional, este grupo no utilizó ningún elemento tecnológico como ayuda adicional a su proceso académico y trabajó de 1 a 2 pm.

El segundo grupo se denominó Grupo Multimedia (M) y trabajó solamente con la multimedia, no hubo mediación de la investigadora (solamente la guía de esta) y trabajó en un laboratorio de cómputo de 2 a 3 pm y finalmente el tercer grupo fue nombrado Grupo Teoría-Multimedia (TM), que trabajó con el método de clase tradicional complementado con la multimedia de 3 a 3:30 pm con teoría y de 3:30 pm a 4 pm con la multimedia. Como se aprecia, el tiempo de trabajo de los tres grupos era el mismo, esto para que la duración de la clase no fuera un factor que afectara los resultados.

Todos los tratamientos fueron supervisados por la investigadora; cabe agregar que los tres grupos asistieron a sus tratamientos una vez por semana los días viernes.

Una vez concluido el período de los tratamientos, se aplicó una entrevista de grupo focal a cada uno de los grupos de tratamiento por separado, cuyo fin era discutir criterios que no eran medibles durante el proceso aplicado a cada grupo de tratamiento que posiblemente 
no se comentaron. Dicha entrevista constaba de cuatro bloques, el primero era del grupo TM el cual tenía seis preguntas, el segundo fue el grupo M con cinco preguntas, el tercero, el grupo T con cuatro preguntas. Para terminar se aplicó un cuarto bloque que constaba de una pregunta general la cual decía la siguiente: “¿Qué otras herramientas utilizarían para mejorar el aprendizaje en la adquisición de destrezas para la evaluación de Componentes de Aptitud Física? ¿Cuántos le agregarían y cuáles agregarían?’. La duración de la entrevista fue de 1 hora por cada grupo de tratamiento y se realizó el día viernes en la tarde en el aula número 2 en CIEMHCAVI. Se formó un círculo en el centro del aula y se colocó la grabadora dentro de este, luego se procedió a iniciar con las preguntas.

Al finalizar el curso se procedió a aplicar el post test de evaluación de componentes de la aptitud física a los tres grupos de tratamiento por separado según a como se habían conformado desde el inicio, para este post test se utilizó el mismo instrumento de evaluación inicial donde se les evaluaría cuánto conocimiento adquirieron en evaluación de componentes de aptitud física y su adecuada ejecución con respecto a la técnica.

Para dar por ganado el curso todos los y las estudiantes, debían cumplir con la evaluación del pre y post test de componentes de aptitud física relacionados a la salud, haber asistido a cada una de las sesiones del tratamiento ya fuese al de T, TM o M; más específicamente la evaluación del curso no era sumativa lo que quiere decir que no era un examen sino una lista de cotejo donde cada ítem correspondía a un componente de aptitud física relacionado con la salud y al concluir con todo esto se les hacía entrega personalmente del certificado con las multimedias de obsequio.

Análisis Estadístico: se aplicó ANOVA de dos vías mixta (3 tratamientos x 2 mediciones) para el análisis cuantitativo y una entrevista de grupo focal para el análisis cualitativo.

Resultados: a continuación se describen los resultados de las estadísticas descriptivas e inferenciales para cada uno de los grupos de tratamiento, obtenidas mediante la evaluación del nivel de destreza de los distintos componentes de aptitud física relacionados con la salud y de las entrevistas de grupo focal.

Para efectos de presentar la información de manera ordenada y secuencial, las estadísticas se presentarán en el orden de las variables dependientes analizadas, los resultados finales obtenidos y las observaciones efectuadas en las entrevistas de grupo focal en los diferentes grupos de trabajo. 


\section{Tabla 1.}

Resultados de evaluación del nivel de destreza en la medición de componentes de aptitud física relacionados a la salud en los diferentes grupos

\begin{tabular}{|l|c|c|c|c|c|c|c|c|}
\hline $\begin{array}{l}\text { Variable } \\
\text { dependiente }\end{array}$ & \multicolumn{2}{|c|}{$\begin{array}{c}\text { Teoría } \\
(\mathrm{n}=9)\end{array}$} & \multicolumn{2}{c|}{$\begin{array}{c}\text { Multimedia } \\
(\mathrm{n}=14)\end{array}$} & \multicolumn{2}{c|}{$\begin{array}{c}\text { Mixto } \\
(\mathrm{n}=6)\end{array}$} & $\begin{array}{c}\text { Total } \\
(\mathrm{n}=29)\end{array}$ \\
\hline & Pre test & Pos test & Pre test & Pos test & Pre test & Pos test & Pre test & Pos test \\
\hline Pliegues cutáneos & $0.50 \pm 0.86$ & $4.19 \pm 1.66$ & $1.41 \pm 1.73$ & $4.50 \pm 1.59$ & $1.33 \pm 1.51$ & $5.25 \pm 1.17$ & $1.11 \pm 1.48$ & $4.56 \pm 1.53$ \\
\hline Fuerza muscular & $1.33 \pm 1.32$ & $3.77 \pm 0.66$ & $0.91 \pm 0.98$ & $3.75 \pm 0.42$ & $2.33 \pm 1.56$ & $3.83 \pm 0.40$ & $1.33 \pm 1.30$ & $3.77 \pm 0.49$ \\
\hline Peso y talla & $2.43 \pm 1.28$ & $3.72 \pm 0.36$ & $1.69 \pm 1.37$ & $3.85 \pm 0.30$ & $2.87 \pm 1.11$ & $3.83 \pm 0.25$ & $2.17 \pm 1.34$ & $3.81 \pm 0.31$ \\
\hline Presión arterial & $1.22 \pm 1.50$ & $3.11 \pm 0.78$ & $1.12 \pm 1.31$ & $3.53 \pm 0.66$ & $1.66 \pm 1.40$ & $3.41 \pm 0.49$ & $1.26 \pm 1.35$ & $3.37 \pm 0.67$ \\
\hline $\begin{array}{l}\text { Capacidad } \\
\text { cardiorrespiratoria }\end{array}$ & $0.88 \pm 1.43$ & $7.61 \pm 0.69$ & $1.41 \pm 1.88$ & $7.46 \pm 0.92$ & $2.37 \pm 2.10$ & $7.41 \pm 0.80$ & $1.44 \pm 1.81$ & $7.50 \pm 0.81$ \\
\hline $\begin{array}{l}\text { Resistencia } \\
\text { muscular }\end{array}$ & $1.24 \pm 1.32$ & $3.72 \pm 0.50$ & $1.46 \pm 1.14$ & $3.46 \pm 1.06$ & $2.29 \pm 1.15$ & $3.41 \pm 1.20$ & $1.56 \pm 1.22$ & $3.53 \pm 0.93$ \\
\hline Flexibilidad & $2.55 \pm 1.59$ & $4.00 \pm 0.00$ & $2.26 \pm 1.39$ & $3.71 \pm 0.57$ & $2.00 \pm 1.51$ & $4.00 \pm 0.00$ & $2.30 \pm 1.44$ & $3.86 \pm 0.42$ \\
\hline Total & $10.18 \pm 6.81$ & $30.13 \pm 2.99$ & $10.28 \pm 5.96$ & $30.28 \pm 4.06$ & $15.20 \pm 8.38$ & $31.16 \pm 3.20$ & $11.27 \pm 6.82$ & $30.42 \pm 3.49$ \\
\hline
\end{tabular}

Nota: se presentan promedios desviaciones estándar

\section{Tabla 2.}

Resumen de ANOVA mixto de dos vías (Mediciones X Grupo) aplicada a los resultados de rendimiento en la evaluación de componentes de aptitud física relacionados a la salud

\begin{tabular}{|l|c|c|c|c|c|c|}
\hline \multirow{2}{*}{ Variable dependiente } & \multicolumn{2}{|c|}{ Mediciones } & \multicolumn{2}{c|}{ Grupo } & \multicolumn{2}{c|}{ Mediciones * grupo } \\
\cline { 2 - 7 } & $\mathrm{F}$ & Sig. & $\mathrm{F}$ & Sig. & $\mathrm{F}$ & Sig. \\
\hline Pliegues cutáneos & 74.3 & $<0.001^{*}$ & 1.5 & 0.23 & 0.416 & 0.66 \\
\hline Fuerza muscular & 74.00 & $<0.001^{*}$ & 2.74 & 0.08 & 2.11 & 0.14 \\
\hline Peso y talla & 33.34 & $<0.001^{*}$ & 1.57 & 0.23 & 2.32 & 0.11 \\
\hline Presión arterial & 47.94 & $<0.001^{*}$ & 0.39 & 0.68 & 0.56 & 0.57 \\
\hline Capacidad cardiorrespiratoria & 262.97 & $<0.001^{*}$ & 0.69 & 0.51 & 1.47 & 0.24 \\
\hline Resistencia muscular & 34.16 & $<0.001^{*}$ & 0.65 & 0.52 & 1.25 & 0.30 \\
\hline Flexibilidad & 30.84 & $<0.001^{*}$ & 0.40 & 0.67 & 0.33 & 7.22 \\
\hline Total & 172.08 & $<0.001^{*}$ & 1.27 & 0.29 & 0.74 & 0.48 \\
\hline
\end{tabular}

*Significativo con $\mathrm{p}<0,01$

En la tabla 1, se pueden observar los promedios y las desviaciones estándar del puntaje obtenido en la evaluación del nivel de aprendizaje en la correcta ejecución de la técnica de pliegues cutáneos en los diferentes grupos. Se realizó análisis de varianza (ANOVA) de dos vías mixta (3 grupos x 2 mediciones), aplicada a los datos de rendimiento que demostraron los y las estudiantes, en su aplicación de la técnica de evaluación de los distintos componentes de aptitud física relacionados a la salud (toma de pliegues cutáneos, valoración de la fuerza muscular, etc.).

Tal y como se aprecia en la tabla 2, en todas las pruebas, se obtuvieron efectos significativos de mediciones, lo cual indica que los y las estudiantes mejoraron significativamente en su rendimiento al aplicar las distintas pruebas. Sin embargo, al no 
existir interacción significativa entre grupo y mediciones las mejoras observadas, no se podrían explicar por diferencias entre las características de los grupos, dado que la mejora en el rendimiento del estudiantado, fue similar en los tres grupos.

\section{Resultados de entrevista de grupo focal}

En la entrevista de grupo focal los resultados de las preguntas fueron los siguientes:

En el bloque de preguntas del grupo $\boldsymbol{T M}$ se presentó una discrepancia en la pregunta número 5, en esta 8 sujetos opinaron en forma positiva pero 3 manifestaron opiniones negativas siendo este el único ítem y grupo en que esto sucedió. Se procedió por tanto a analizar esas tres opiniones que fueron las siguientes: 1) Se está más acostumbrado al trabajo con la clase tradicional y material impreso, 2) consideran que ambos son métodos que requieren de cierto manejo de conocimientos y 3) es difícil medir el nivel de aprendizaje en ambos métodos cuando no se ha contado con antecedentes del uso de estas nuevas herramientas de enseñanza a nivel universitario en CIEMHCAVI.

Ahora bien, entre las opiniones positivas obtenidas en la entrevista de grupo focal para el grupo TM, en general los estudiantes expresaron que la multimedia es mejor que recibir una clase tradicional porque facilita navegar a través de la teoría y se logra un mayor aprendizaje observando videos. También refuerza la teoría, porque con la lectura se empieza a crear la imagen visual mientras que la multimedia la muestra.

Además, es un material bonito, distinto, innovador y llamativo, de fácil ejecución, el cual provoca una interacción e intercambio de información recibida por el estudiante y enviada por la multimedia. Permite a su vez un aprendizaje individual en la teoría y grupal en la práctica. Si no se recuerda algo la multimedia permite buscarlo y recordarlo. Es más atractiva en elementos visuales y si algún contenido no queda claro con la teoría, el video permite evacuar las dudas.

La combinación de medios le permite al estudiante llegar a su casa y repasar la materia con la multimedia. No limita al estudiante a solo leer sino a poder navegar en la multimedia e iniciar donde los estudiantes quieran o necesiten aplicar en ese momento.

Es una herramienta útil para aprender con facilidad, que permite repasar tanto la teoría como la práctica donde no se requiere la presencia de un experto en la materia sino de un guía porque la información de la multimedia es muy explícita y por tanto no deja dudas. Ofrece una retroalimentación y la posibilidad de tener el material impreso que se necesite en ese momento. 
Con respecto a sus posibles aplicaciones dentro de la academia, este tipo de herramienta debería ser incluida dentro de los planes de estudio de las carreras de la CIEMHCAVI donde sean utilizadas para reforzar ciertos cursos de la misma.

Esta es una herramienta que forma parte de los avances y beneficios que ofrece la tecnología, como la facilidad de obtener información más seleccionada y a bajo costo.

Se recomienda para todas aquellas personas que no tengan acceso a una biblioteca o internet porque el disco almacena la información necesaria que permite desarrollar la labor docente con eficacia y precisión.

Por su parte, en el grupo $\boldsymbol{M}$ las preguntas fueron contestadas $100 \%$ en forma positiva; en este bloque los estudiantes manifestaron que la multimedia permite una fácil navegación de un concepto a otro, el video permite verlo una y otra vez, aclara dudas en relación con la ejecución de la técnica correcta para la evaluación de los componentes de aptitud física relacionados con la salud. Le permite al estudiante ser autodidacta, le da posibilidad de que no pierda ninguna clase y en el caso del profesor este puede falta a la lección permitiéndole después aclarar dudas solamente.

En el grupo $\boldsymbol{T}$, en las preguntas de la número uno a la cuatro los estudiantes responden en un $100 \%$ de forma favorable y en sus comentarios indican que se refuerza mucho la teoría con la práctica y que se toman en cuenta los puntos necesarios, rescatando lo más importante de cada uno de los protocolos de evaluación de los componentes de aptitud física relacionados con la salud. Si bien lo anterior muestra cierta tendencia a que solo la teoría es positiva, los mismos estudiantes indicaron que el trabajo con sólo teoría es muy agotador por lo que se necesita agregar otros tipos de medios que faciliten el aprendizaje.

\section{Discusión:}

El objetivo de este estudio fue determinar el nivel de aprendizaje académico de componentes para la evaluación de la aptitud física relacionada a la salud, en un grupo de estudiantes que cursa la carrera de educación física mediante la utilización de una multimedia.

Los resultados mostraron en general que no hubo interacción significativa entre mediciones y grupos en ninguna de las variables evaluadas (Porcentaje de grasa, 1 RM y 8 RM, Resistencia muscular, Flexibilidad, Capacidad Cardiorrespiratoria, peso, talla, presión arterial y puntaje total); asimismo, no hubo diferencias significativas entre grupos en ninguna de las variables. 
Si bien, en algunos análisis se observa una tendencia a que el grupo mixto (TM), tenía mayores puntajes que los grupos de solo multimedia (M) y solo teoría (T), esto probablemente ocurrió debido a que las muestras de los sujetos en los grupos eran pequeñas por una parte $[10(\mathrm{~T}), 14(\mathrm{M}), 6(\mathrm{TM})]$, pero por otro lado, los resultados muestran que esas tendencias eran relativamente pequeñas para provocar interacciones significativas y que además, al evolucionar de una medición a otra de manera similar, hizo que no se obtuvieran diferencias significativas.

Por otro lado, las mediciones pre y pos test si presentan una diferencia significativa, lo anterior es interesante, pues muestra que los tratamientos fueron los que provocaron cambios en sus respectivos grupos, lo cual indica que estos fueron lo suficientemente efectivos para mejorar las destrezas para la evaluación de componentes de aptitud física relacionados con la salud. Más aún, los mismos resultados mostraron que entre los grupos no solo no se dieron cambios significativos, sino que en algunas variables evaluadas, los estudiantes que recibieron TM y solo M, obtuvieron una nota ligeramente más alta que los estudiantes del grupo T, lo que permite mostrar que hubo un aprendizaje importante con el uso de la multimedia.

Del mismo modo, parte de los cursos que son impartidos en CIEMHCAVI son de modalidad teórico - práctico, por lo que los estudiantes necesitan realizar diferentes prácticas para poder incrementar su nivel de destrezas en sus respectivos cursos; dado que la mayoría del material bibliográfico se basa en material impreso y no digital, no le permite a los estudiantes observar y analizar esas destrezas en tiempo real pues el material impreso viene con fotografías, dibujos y esquemas, mientras que la multimedia ofrece videos, sonido, imágenes estáticas y en movimiento, por lo que los estudiantes podrán apreciar la ejecución de todo este conjunto de destrezas en el mismo instante en que las ponen en práctica.

Esto último se ve reforzado por Gándara, 1997; Josemaría, 2001; Salinas, 2004 y Sánchez, 2003, quienes indican que entre las múltiples ventajas de la multimedia se encuentra que son agradables en lo que respecta al plano visual, es un material interactivo el cual estimula el aprendizaje individual, además, al involucrar diferentes medios tecnológicos permite potencializar de distintas maneras los procesos de aprendizaje de los estudiantes y por ende, los procesos pedagógicos.

En cuanto a las tendencias encontradas, en la entrevista de grupo focal los estudiantes mencionaban que en el caso del grupo de teoría, la experiencia fue muy provechosa y el conocimiento bien aplicado dado que la práctica les facilitó el aprendizaje de los contenidos del curso, pero en el grupo de multimedia y en el de multimedia - teoría, la experiencia fue distinta pero también de mucho beneficio, debido a que el trabajo con la multimedia les permitía a los estudiantes consultar cada uno de los protocolos las veces que fuera necesario y además el poder aprender a través de distintos medios como el video, audio, la escritura y tener la posibilidad de realizar una autoevaluación después de cada protocolo, aparte de la facilidad de navegar a través de la teoría, con la que se aprende más observando el ejemplo 
en video y al mezclar lo teórico y lo audiovisual, es más fácil aprender, lo que se ve reforzado por la teoría.

Estas opiniones son importantes porque todas se dirigen a procurar un mejoramiento de la calidad de los procesos académicos que se trabajan en la CIEMHCAVI, tomando en cuenta para esto que debe existir la posibilidad y con ello la obligación de que los docentes estén conscientes de que deben de actualizarse y hacer un uso adecuado de dichas herramientas de aprendizaje y así contribuir a mejorar la educación de la CIEMHCAVI y de la Universidad en general. Propiamente, los resultados obtenidos permiten asegurar que mediante esta herramienta tecnológica, los estudiantes y profesionales egresados de la CIEMHCAVI puedan obtener los conocimientos necesarios en lo referente no solo al campo de la medición y evaluación de componentes de aptitud física relacionados con la salud, sino que también, con todas las demás áreas del movimiento humano y la salud y por ende mantenerse actualizados.

Otro de los aspectos que muestra nuevamente las fortalezas de este método es su relativo bajo costo, pues toda la información necesaria se almacena en un disco compacto, por lo que los estudiantes y profesionales que vivan lejos de los centros educativos podrán disponer de este material y revisarlo en sus hogares, en un café internet o algún lugar donde cuenten con una computadora y por lo tanto evitar desactualizarse.

\section{CONCLUSIONES:}

La entrevista de grupo focal, permitió detectar que el estudiantado de los grupos que recibieron el curso con multimedia (solo multimedia y mitad multimedia y mitad teoría), tuvo opiniones favorables con respecto a la utilización y aplicabilidad de este medio, como herramienta de aprendizaje, en las temáticas del curso de Evaluación de Componentes de Aptitud Física relacionados con la salud.

Además, fue claro en las opiniones del estudiantado que recibió un curso teórico, que esta modalidad es poco estimulante o motivante, aunque se puede igual aprender en un curso así.

Y en general, el estudiantado de los tres grupos, coincide en la importancia de la utilización de herramientas tecnológicas para mejorar los procesos académicos a nivel de la educación superior costarricense.

\section{RECOMENDACIONES:}

Al diseñar una multimedia para uso educativo se debe dar gran importancia a criterios como la interactividad, la calidad de los medios, la estructura de navegación y otros que pueden afectar su utilidad. 


\section{REFERENCIAS:}

Álvarez, C y Hernández, R. (2005). Pasos adelante del determinismo educativo: Ambientes colaborativos virtuales de aprendizaje. Programa Novus - Universidad Nacional. Heredia, Costa Rica: Programa Novus.

Gándara, M. (1997). ¿Qué son los programas multimedios de aplicación educativa y como se usan? ULSA, México. Recuperado desde: http://redescolar.ilce.edu.mx/redescolar/biblioteca/articulos/pdf/S\%EDntesis\%20G\%E1 ndara.pdf.

Josemaría, C. (2001). Diseño de multimedias educativas. Criterios didácticos y selección de multimedias educativas. Buenos Aires, Argentina. Recuperado desde: www.librosenred.com.

Salinas, J. (2004). Multimedia en los procesos de enseñanza-aprendizaje: elementos de discusión. Universidad de las Islas Baleares, España. Recuperado desde: http://www.uib.es/depart/gte/edutec99.html.

Sánchez, J. (2003). Producción de aplicaciones multimedias por docentes. Universidad de Málaga, España. Revista de Medios y Educación, 21(Julio), 85-98.

Sierra, F. (2002). La Educación superior y los tipos de multimedia de interacción simbólica. Universidad de Sevilla, España. Recuperado desde: http://usuarios.trcnet.com.ar/denise/repositorio/NTICs_y_EducacionSuperior.pdf.

Fecha de recepción: 29 de marzo de 2012.

Fecha de aceptación: 4 de junio de 2012.

Fecha de publicación: 31 de julio del 2012. 